\title{
Dependencia-Independencia de Campo y Diferenciación Hemisférica en tareas de escucha dicótica
}

\section{Rocio Fernández-Ballesteros y Lilianne Manning Melean}

Universidad Autonoma de Madrid

\section{INTRODUCCION}

La asociación entre la DependenciaIndependencia de Campo (DIC) y la Diferenciación Hemisférica (DH) ha sido postulada por Witkin, Goodenough $y$ Oltman (1979). Esta asociación, basada en la Teoria de la diferenciación de Witkin - la cual supone tanto una diferenciación psicológica como neurológicaha recibido en los últimos años un importante aval empírico.

La hipótesis general de esta relación entre la DIC y la diferenciación hemisférica, no implica, desde luego, una mayor diferenciación en uno de los hemisferios (lo que daria consigo una tendencia hemisférica), sino que postula una mayor especialización de cada hemisferio en el procesamiento de las tareas para las cuales se hallan especializados. Como se sabe, en sujetos diestros, el hemisferio izquierdo se ha considerado como mediador de los procesos verbales y analíticos mientras que el derecho parece tener una función eminentemente visoespacial.

Kimura (1979) realiza una sintesis de las distintas tareas que son procesadas fundamentalmente por uno $\mathrm{u}$ otro hemisferio atendiendo a la modalidad sensorial del input. Así, aporta evidencia acumulativa por la cual el procesamiento visual de letras, sílabas y palabras corresponde al hemisferio izquierdo mientras que el procesamiento de información visual conteniendo puntos en dos dimensiones, enumeración de puntos y formas, inclinación de líneas así como profundidad, se produce en el hemisferio derecho. Con respecto a la modalidad manual, los movimientos que requieren precisión así como los movimientos espontáneos al mantener una conversación parecen estar lateralizados en el hemisferio izquierdo mientras que existe escasa evidencia respecto a la especialización del hemisferio derecho en el procesamiento de inputs táctiles. Por lo que se refiere a la modalidad auditiva, palabras, sílabas sin sentido e, incluso, la audición de conversaciones invertidas son procesadas en el hemisferio izquierdo mientras modelos melódicos y sonidos no-lingüísticos lo son en el hemisferio derecho.

Hasta la fecha se han establecido relaciones consistentemente significativas en- 
tre la DIC y la DH operativizando ésta mediante pruebas de modalidad visual. Así, Zoccolotti y Oltman (1978) a través de tareas de reconocimiento de letras y caras concluyeron que los sujetos Independientes de Campo (IC) mostraban una diferencia significativa a favor de la discriminación de letras presentadas en el campo visual derecho ( $y$, por tanto proyectadas en el hemisferio izquierdo) y de caras presentadas en el campo visual izquierdo (y por tanto proyectadas en el hemisferio derecho) mientras que en los sujetos Dependientes de Campo (DC) no se apreciaban diferencias significativas entre ambas tareas y su proyección a uno u otro hemisferio. En este mismo sentido, Pizzamiglio y Zoccolotti (1981) estudiaron la influencia de la DIC en el procesamiento de dos tipos de tareas una verbal y otra de reconocimiento de caras; los resultados evidenciaron que los sujetos IC presentaban significativamente mejores ejecuciones cuando los estímulos verbales les eran proyectados en el hemisferio izquierdo (enviados a través del hemicampo derecho) y cuando las caras eran proyectadas en el hemisferio derecho (habiendo sido enviadas por el hemicampo izquierdo). Por el contrario, no existian diferencias significativas en la ejecución de los sujetos DC en función del hemisferio en el que los estimulos eran proyectados. Oltman, Ehrlichman y Cox (1977) utilizando una tarea de percepción de caras encontraron que los sujetos IC procesaban mejor esta tarea en el hemisferio derecho que en el izquierdo mientras que en los sujetos DC no existian diferencias significativas entre el procesamiento en el hemisferio derecho o izquierdo. En esta misma línea de investigación Fernández-Ballesteros y Manning (1981) encontraron que los sujetos IC ejecutaban significativamente mejor una tarea de reproducción de puntos distribuidos en el espacio cuando los estimulos eran enviados mediante tasquistoscopio desde el hemicampo izquierdo del sujeto ( $y$, por tanto, eran proyectados en su hemisferio derecho) en comparación a cuando eran enviados desde el hemicampo derecho, mientras que no existian diferencias significativas en la ejecución de los sujetos DC en ambas condiciones. Por otra parte, Manning y Fernández-Ballesteros (1982) pusieron en evidencia que en una tarea de reproducción de letras los sujetos IC rendian significativamente mejor cuando los estímulos verbales eran presentados en el hemicampo derecho (proyectados en el hemisferio izquierdo) que cuando se les presentaban en el izquierdo, no existiendo diferencias significativas en los sujetos DC. Por último, Pizzamiglio et al. (1983) y Manning (en prensa) han puesto de manifiesto cómo los sujetos IC ejecutan mejor una tarea de reconocimiento de expresiones faciales presentadas mediante tasquitoscopio en uno $\mathbf{u}$ otro hemicampo visual, cuando éstas eran procesadas en el hemisferio derecho $y$, por tanto, fueron recibidas a través del hemicampo visual izquierdo.

Si tras la revisión efectuada puede concluirse, que en efecto, existe una relación consistente entre la DIC y la DH en el sentido de que mientras que los sujetos IC presentan una marcada especialización hemisférica no ocurre así en los DC, esta conclusión es válida cuando se operativiza la DH mediante tareas visuales. Cabe preguntarse si esta evidencia es igualmente concluyente cuando se utilizan inputs táctiles o auditivos. Tal pregunta no es baladí por cuanto la DIC es generalmente operativizada mediante tareas también visuales y ateniéndonos a la evidencia suministrada no cabría realizar generalizaciones sobre la relación que nos ocupa cuando como medida de la DH se utilizan otras tareas no visuales.

La realidad es que existen muy pocas investigaciones que pongan en relación estos dos constructos - la DIC y la $\mathrm{DH}$ - operativizando esta última mediante ejecuciones en tareas táctiles y auditivas.

Con respecto a la modalidad táctil, si bien Zoccolotti, Passafiume y Pizzamiglio (1979) lograron detectar diferencias significativas entre sujetos DC e IC en una tarea táctil de orientación de líneas, 
Manning y Fernández-Ballesteros (1985) no obtuvieron los mismos resultados en una de reconocimiento de formas. Estos resultados incongruentes, van en la misma linea señalada por numerosos autores sobre la precaución con la que deben ser utilizados estímulos táctiles en tareas de medida de la DH (Benton, Levin y Barney, 1973; Benton, Barney y Hamsher, 1978; Beaumont, 1983).

Por lo que se refiere a la modalidad auditiva, y ya que está bien establecido en la literatura general sobre el tema que el material auditivo verbal es prioritariamente procesado en el hemisferio izquierdo mientras que el musical lo es en el derecho (ver, entre otros, Milner 1962; Kimura 1961 a, b, 1964, 1979; Gordon 1974, 1983), parecería lógico que si, en efecto, existiera una estrecha relación entre la DIC y la $\mathrm{DH}$, utilizando el procedimiento de la escucha dicótica, los sujetos IC (controlando, por supuesto sus capacidades auditivas) deberían procesar diferenciadamente el material auditivo verbal mejor por el canal derecho (el cual sería proyectado al hemisferio izquierdo) y el musical por el izquierdo (proyección hemisferio derecho) mientras que en los sujetos DC no aparecería este tipo de especialización.

Sin embargo, como ya se anotó, existe muy escasa investigación en este sentido. En efecto, tan sólo hemos encontrado un trabajo referido al tema en el que Pizzamiglio (1974) estudió la lateralidad manual de sujetos DC e IC utilizando también una prueba de escucha dicótica. Planteó la hipótesis de que los sujetos totalmente diestros y que evidenciaran una clara preferencia del oído derecho en la prueba de escucha dicótica con material verbal, presentarian una más alta puntuación en IC que aquellos que no presentaran ventaja a favor de uno u otro oído y-o fueran ambidextros. Por lo que se refiere a la prueba de escucha dicótica, el autor dividió a los sujetos en dos grupos, partiendo de la proporción de errores cometidos en la escucha a través del oído izquierdo frente a errores en el derecho. Operativizando la DIC mediante el EFT ( Embbeded Figure Test») el RFT («Rod-and-Frame Test») y el DAP («Draw-a-Person») concluyó que los sujetos IC presentaban mejores ejecuciones cuando el material verbal era enviado a través del oído derecho (proyección en el hemisferio izquierdo).

A pesar de la escasa evidencia empirica existente o, tal vez por ello, nos planteamos comprobar la posible relación entre la DIC y la DH operativizando ésta mediante la ejecución de los sujetos en pruebas de escucha dicótica de material verbal y musical. El supuesto básico es el siguiente: los sujetos IC presentarán en tareas de escucha dicótica verbal mejores ejecuciones cuando los estímulos sean presentados a través del canal derecho (con proyección en el hemisferio izquierdo) mientras que no existirán diferencias significativas entre ambos canales en sujetos DC. Asimismo, los sujetos IC presentarán mejores rendimientos en tareas musicales de escucha dicótica en el canal izquierdo (con proyección en el hemisferio derecho) mientras que los sujetos DC no presentarán ejecuciones diferenciales en ambos canales y hemisferios.

No se nos oculta que tales hipótesis plantean problemas metodológicos tanto por lo que se refiere a la operativización de la dimensión DIC como en el establecimiento de las pruebas de escucha dicótica. En distintos trabajos (ver Fernández-Ballesteros 1980, Fernández-Ballesteros y Manning 1981, Manning y Fernández-Ballesteros 1982, Fernández-Ballesteros y Manning 1985) nos hemos referido a las distintas medidas de la DIC y a la necesidad de utilizar distintos instrumentos a la hora de operativizar este constructo así como a la necesidad de establecer un procedimiento a través del cual comprobar la validez de constructo de esta dimensión y poder así proceder a la selección de sujetos DC e IC. Por otra parte, existe también evidencia de la cautela y el cuidado con el que conviene proceder a la hora de construir y utilizar pruebas de escucha dicótica en la evaluación de la DH (para una revisión ver Beaumont 1983). Teniendo 
en cuenta todo ello, abordamos la presente investigación.

\section{METODO}

\section{Sujetos}

Se obtuvo una muestra de 60 sujetos (30 varones y 30 mujeres, edades comprendidas entre 17 y 38 años con una edad promedio de 22,3 años) voluntarios estudiantes universitarios de la Facultad de Psicología de la Universidad Autónoma de Madrid. Los sujetos fueron clasificados en 27 dependientes (DC) y 33 independientes de campo (IC). Todos los sujetos eran diestros y no presentaban problemas auditivos.

Esta muestra fue seleccionada a partir de un grupo de 250 sujetos voluntarios, 100 varones y 150 mujeres en función de sus puntuaciones en las siguientes medidas de la DIC: RFT («Rod-and-Frame Test»), EFT («Embedded Figure Test») y GEFT ( Group Embedded Figure Test»). El procedimiento establecido para la clasificación de los sujetos en DC e IC así como los datos correlacionales y factoriales sobre la validez de contructo de la operativización de la DIC utilizada figuran en el trabajo de Fernández-Ballesteros y Manning (1985) y Manning, Fernández-Ballesteros y Maciá (en prensa).

\section{Instrumentos}

Prueba de Escucha Dicótica verbal: Fue grabada una cinta magnetofónica de dos canales a través de un magnetófono marca «Grundig», modelo TK 46, estereofónico, con 4 pistas de grabación independientes el cual disponía de efecto "play-back", sobre-grabación y analizador de volumen por canal.

Se grabaron dos tipos de elementos verbales, dígitos y palabras monosilábicas en pares de la misma entidad y características. El total de dígitos grabados fue de 16 y asimismo el total de palabras fue de 16 . Se agruparon cuatro elementos por cada canal para cada ensayo. El total de agrupaciones fue de 8 . El total de elementos grabados de 64 y el total de elementos preparados para ser recibidos por cada sujeto fue de 128 . Los intervalos interestimulares de 1 segundo y los intervalos intergrupos de 10 segundos. Se estableció una presentación aleatorizada de números y letras por bloques de ensayo.

El instrumento quedó así constituido tras un estudio piloto en el que fueron testados 12 sujetos y en el que se perfilaron algunos de los elementos iniciales.

Prueba de Escucha Dicótica musical: Se utilizó la cinta grabada con las melodias, en dos canales, original de Kimura ("dk Consultants», Ontario Canadá, 1979). Consta de 14 fragmentos melódicos de Vivaldi, Mozart, Teleman, Bach y Antonioni, interpretados en su mayoría con instrumentos de viento. Cada uno de los 14 «sets» de la prueba, consta de una primera emisión dicótica, es decir, dos fragmentos musicales diferentes, uno por cada canal grabados simultáneamente, se produce entonces un intervalo de 4 segundos y luego cuatro emisiones biaurales con 3 segundos de intervalo entre ellas. Entre estas cuatro melodías se encuentran las dos inicialmente recibidas.

Este instrumento fue puesto a punto mediante un estudio piloto, con 12 sujetos, con el fin de adaptar las consignas que deberían darse a los sujetos de la muestra.

El aparato utilizado para ambas Pruebas Dicóticas fue un magnetófono marca «Sony» con un «set» de audifonos directamente incorporados.

\section{Procedimiento}

El experimento fue realizado en el $\mathrm{La}$ boratorio del Departamento de Diagnóstico Psicológico y Medida de la Universidad Autónoma de Madrid, manteniéndose las condiciones ambientales óptimas en cuanto a ruido y temperatura. 


\section{Estudios}

Para aplicar la Prueba Dicótica Verbal, se explicaba al sujeto que iba a recibir 4 dígitos o palabras cortas por un oído y otras 4 por el otro en forma simultánea. La tarea que se le demandaba era decir en voz alta todos los estímulos que recordara durante el espacio de pausa interbloques. Se le advertía que no se preocupara por el orden de recepción de los estímulos ni por lograr un recuerdo exhaustivo de los mismos.

La mitad de la muestra empezó ambas Pruebas con los audífonos en posición «normal»y una vez terminada la grabación, se cambiaba a la posición «inversa», de manera que al finalizar la prueba, ambos oidos habían recibido todos los elementos. Para la otra mitad de la muestra se actuó en sentido inverso. El volumen del aparato fue adaptado a cada sujeto y este paso se efectuó durante el anuncio de la prueba que iba a recibir.

La puntuación que fue asignada a cada sujeto en la Prueba Dicótica Verbal implicaba la suma de elementos recordados apropiadamente por cada canal auditivo. La puntuación de la Prueba Dicótica Musical' consistió en el número de aciertos en uno y otro canal auditivo.

El orden de aplicación de las pruebas fue contrabalanceado.

\section{Tratamiento estadístico de los datos}

Puesto que, en ambos casos, las puntuaciones obtenidas eran discretas fueron transformadas en puntuaciones lineales por la fórmula de Ascombe. Con estas puntuaciones, para cada oído, fueron calculadas las Medias aritméticas y las desviaciones típicas tanto para el grupo total como para los dos grupos de sujetos que habían sido clasificados según la DIC en dependientes (DC) e independientes de campo (IC). Se aplicó la prueba «t» de Student para conocer la significación de diferencias entre medias de los tres grupos: total, DC e IC (estos dos últimos según sus puntuaciones ponderadas), en la ejecución de estímulos auditivos entre oído izquierdo y derecho. Todo ello, para las dos Pruebas.

\section{RESULTADOS}

Los resultados que se presentan en el Cuadro 1 corresponden a los hallados para la Prueba Dicótica Verbal, en él puede apreciarse que existe una diferencia altamente significativa en las puntuaciones entre oídos tanto en el grupo total como en el grupo clasificado como IC, a favor del oído derecho (total: $\mathrm{t}=$ $6.56, \mathrm{p}<.001 ; \mathrm{IC}: \mathrm{t}=10.1, \mathrm{p}<.001)$. Por otra parte en este Cuadro se reflejan las medias aritméticas muy cercanas para cada canal auditivo para los sujetos DC que, por tanto, no obtienen diferencias significativas.

\section{CUADRO 1}

Escucha Dicótica Verbal

Medias aritméticas, D.T. y $t$ de Student: Grupos Total - I.C. - y D.C.

\begin{tabular}{|c|c|c|c|c|c|c|c|c|c|c|c|}
\hline \multicolumn{4}{|c|}{ Total } & \multicolumn{4}{|c|}{ I.C. } & \multicolumn{4}{|c|}{ D.c. } \\
\hline \multicolumn{4}{|c|}{$N=60$} & \multicolumn{4}{|c|}{$\mathbf{N}=33$} & \multicolumn{4}{|c|}{$N=27$} \\
\hline \multicolumn{2}{|c|}{0.1.} & \multicolumn{2}{|c|}{ O.D. } & \multicolumn{2}{|c|}{ 0.1. } & \multicolumn{2}{|c|}{ O.D. } & \multicolumn{2}{|c|}{0.1.} & \multicolumn{2}{|c|}{ O.D. } \\
\hline$\dot{\mathbf{x}}$ & D.T. & $\bar{x}$ & D.T. & $\bar{x}$ & D.T. & $\overline{\mathbf{x}}$ & D.T. & $\overline{\mathbf{x}}$ & D.T. & $\overline{\bar{x}}$ & D.T. \\
\hline \multirow[t]{2}{*}{5,86} & 0,6 & 6,48 & 0.54 & 5,65 & 0,6 & 6,7 & 0,47 & 6,08 & 0,51 & 6,21 & 0,48 \\
\hline & \multicolumn{3}{|c|}{$t=6,56^{* * *}$} & \multicolumn{4}{|c|}{$t=10,1^{* *}$} & \multicolumn{4}{|c|}{$t=1,16$} \\
\hline
\end{tabular}

$\cdots p<0,001$. 
En el Cuadro 2 se presentan los resultados para la Prueba Dicótica Musical. En él pueden apreciarse las diferencias altamente significativas $(\mathrm{p}<.001)$ para la muestra total a favor del oido izquierdo $(t=6.81)$. Igualmente ocurre esto en los sujetos IC con una «t» aun de mayor magnitud $(t=10.04)$. Frente a ello, el grupo de sujetos DC no alcanzan significación estadística en la diferencia de aciertos entre canales auditivos.

\section{CUADRO 2}

Escucha Dicótica Musical

Medias aritméticas, D.T. y $t$ de Student: Grupos Total - I.C. - y D.C.

\begin{tabular}{|c|c|c|c|c|c|c|c|c|c|c|c|}
\hline \multicolumn{4}{|c|}{ Total } & \multicolumn{4}{|c|}{ I.c. } & \multicolumn{4}{|c|}{ D.C. } \\
\hline \multicolumn{4}{|c|}{$\mathbf{N}=60$} & \multicolumn{4}{|c|}{$\mathbf{N}=\mathbf{3 3}$} & \multicolumn{4}{|c|}{$N=27$} \\
\hline \multicolumn{2}{|c|}{0.1.} & \multicolumn{2}{|c|}{ O.D. } & \multicolumn{2}{|c|}{ 0.I. } & \multicolumn{2}{|c|}{ O.D. } & \multicolumn{2}{|c|}{0.1.} & \multicolumn{2}{|c|}{ O.D. } \\
\hline $\bar{x}$ & D.T. & $\bar{x}$ & D.T. & $\bar{x}$ & D.T. & $\bar{x}$ & D.T. & $\overline{\mathbf{x}}$ & D.T. & $\bar{x}$ & D.T. \\
\hline 4,5 & 0,4 & 3,9 & 0,4 & 4,7 & 0,3 & 3,99 & 0,5 & 4,2 & 0,4 & 4,1 & 0,3 \\
\hline \multicolumn{4}{|c|}{$t=6,81^{* * *}$} & \multicolumn{4}{|c|}{$t=10,04 * * *$} & \multicolumn{4}{|c|}{$t=1,35$} \\
\hline
\end{tabular}

$\cdots p<0,001$.

\section{DISCUSION}

Los resultados obtenidos relativos a la Prueba de Escucha Dicótica Verbal referidos a la muestra total van en la misma línea de los hallazgos generales sobre diferenciación hemisférica operativizada a través de escucha dicótica con estímulos verbales, a saber, que los sujetos presen$\tan$ mejores ejecuciones en la información verbal (simultáneamente presentada por ambos canales auditivos) que escuchan por el canal auditivo derecho por lo que es de suponer que esta tarea se está procesando en el hemisferio izquierdo (Milner 1962, Kimura 1961 a, b, 1964, 1973; Gordon 1974, 1983, entre otros).

Por otra parte, se contrasta nuestra hipótesis que postulaba que los sujetos diferirian según su DIC en el sentido de que los sujetos IC percibirian mejor los elementos verbales emitidos a través del canal derecho y que, por tanto, procesarian la información en el hemisferio izquierdo mientras que los sujetos DC no presentarian diferencias en razón a la emisión en uno u otro canal auditivo.
Esto va en la misma línea de lo hallado por Pizzamiglio (1974). Es decir, los sujetos IC manifestarian mayor grado de diferenciación a la hora de procesar una información verbal frente a los sujetos DC.

Asimismo, los resultados obtenidos referidos a la Prueba Dicótica Musical para la muestra total ponen de manifiesto el procesamiento de la información musical (del tipo de la utilizada por nosotros) en el hemisferio derecho, esto es coherente con los resultados obtenidos desde la investigación básica en diferenciación hemisférica (para una revisión ver Gordon 1983).

Por otra parte, hemos contrastado nuestra hipótesis, es decir, los sujetos IC procesan la información de tipo melódico en el hemisferio derecho mientras que los sujetos DC no presentan tal diferenciación hemisférica. Esto parece un nuevo hallazgo en la relación de la DIC con el procesamiento melódico ya que no existen -en nuestro conocimientodatos publicados al respecto. 


\section{CONCLUSIONES}

Los resultados procedentes de los dos experimentos realizados ponen de relieve los siguientes hallazgos:

1) Se contrastan todos aquellos resultados procedentes de la investigación básica en diferenciación hemisférica por los cuales puede afirmarse a altos niveles de probabilidad que cuando son enviados estímulos auditivos verbales mediante escucha dicótica éstos son procesados fundamentalmente en el hemisferio izquierdo. $\mathrm{Y}$, asimismo, cuando son emitidas dicóticamente melodías (del tipo empleado en el experimento 2) éstas son procesadas en el hemisferio derecho.

2) Se han verificado también a altos niveles de probabilidad las dos hipótesis planteadas. Los sujetos independientes de campo procesan la información verbal enviada simultáneamente a ambos canales auditivos significativamente mejor en el hemisferio izquierdo que en el derecho mientras que cuando se emiten melodías mediante escucha dicótica éstas son procesadas en el hemisferio derecho. Por el contrario, los sujetos dependientes de campo no presentan esta diferenciación al procesar ni la información auditiva verbal ni la musical. Este hallazgo es especialmente importante puesto que el tipo de prueba a través del cual se ha evaluado la diferenciación hemisférica implica una modalidad sensorial diferente (auditiva) al de los instrumentos mediante los cuales los sujetos han sido seleccionados en la dimensión dependencia-independencia de campo (fundamentalmente visual). Por tanto, puede suponerse que la relación entre la dependencia-independencia de campo con la diferenciación o especialización hemisférica no se reduce al procesamiento de estímulos visuales y que, por tanto, existe una cierta generalización en tal relación.

3) Por último, cabe señalar la importancia de ciertos constructos psicológicos, como es el caso de la dependenciaindependencia de campo, en la modulación de los principios generales procedentes de la investigación básica en diferenciación hemisférica.

\section{Resumen}

En una muestra de 60 estudiantes voluntarios, se ba examinado la relación existente entre el estilo perceptivo "dependencia-independencia de campo» medido a través del RFT, EFT y GEFT y el grado de diferenciación bemisférica auditiva operativizado mediante dos tareas de escucha dicótica, una verbal y otra musical. Los resultados revelan un procesamiento claramente lateralizado en los sujetos independientes de campo frente a una bilateralización en los dependientes de campo.

\section{Summary}

Relationships, at a bighly significant level, bave been found between, in one hand fiel dependence-independence as measured by three task (RFT, EFT and GEFT) and, in the other hand, auditory lateralization assessed by dichotic listening in two different situations according to the stimuli: verbal and musical presentations, in a sample of 60 volunteers college students.

\section{Résumé}

Sur un echantillon de 60 etudiants volúntaires, on a etudie la relation existant entre le style perceptif dépendence-indépendence à l'égard du champs, mesuré à l'aide de trois tâches visulles (RFT, EFT et GEFT), et le degré de lateralization bémisphérique auditive, evalué au moyen de deux tâches d'écoute dichotiaue, l'une verbale et l'autre musicale. Les resultats montrèrent un plus baut degré de differentiation chez les sujects indépendents à l'egard du champs. 


\section{Referencias}

BeAumont, J. G.: «Methods for studying cerebral hemispheric function». En A. W. Young (Ed.) Funttions of the Right Cerebral Hemisphere. Academic Press, London, 1983.

Benton, A.) VARNeY, N., y HAMSHER, S.: «Lateral differences in tactil directional perception». Neuropsychologia, 1978, 16, 109-114.

Benton, A.; LeVIN, H., y VARney, N.: "Tactil perception of direction in normal subjects». Neurology, $1973,23,1248-1250$.

FERNÁNDEZ-BALlESTFROS, R.: «Del estilo cognitivo “dependencia-independencia de campo" a una teoria de la diferenciación». Revista de Psicologia General y Aplicada, 1980, 35, 467-490.

FERnÁndez-BALlesteros, R., y MANNING, L.: «Dependencia-independencia de campo y diferenciación hemisférica. 1. Asimetría derecha en una tarea de localización espacialm. Revista de Psicologia General y Aplicada, 1981, 36, 3, 385-392.

FERNÁndez-BALlesteros, R., y MANNing-MEleAN, L.: «Diferenciación hemisférica, procesamiento de la información y estilo cognitivo». En J. Mayor (Ed.) Actividad Humana y Procesos Cognitivos. Editorial Alhambra, S.A. Madrid, 1985.

GoRDON, H.: «Auditory specialization of the right and left hemispheres». En M. Kinsbourne y W. Smith (Eds.) Hemispheric Disconnection and Cerebral Function, Springfield Ill. Thomas, 1974.

Gordon, H.: «Music and the right hemisphere». En A. W. Young (ed.) Functions of the Right Cerebral Hemisphere. Academic Press, London, 1983.

KImURA, D.: «Cerebral dominance and the perception of verbal stimuli». Canadian Journal of Psycbology, 1961, a, 15, 166-171.

Kimura, D.: "Some effects of temporal lobe damage on auditory perception». Canadian Journal of Psychology, 1961b, 15, 156-165.

KimURA, D.: «Left-right differences in perception of melodies». Quarterly Journal of Experimental Psychology, $1964,14,355-358$.

Kimura, D.: «La asimetría del cerebro humano». En Psicología Fisiológica, Blume Ediciones, Madrid, 1979.

MANNING, L.: «Interhemispheric asymmetry in facial expression recognition: Relationship to field dependence». En prensa.

MANning, L., y Fernández-Ballesteros, R.: «Dependencia-independencia de campo y diferenciación hemisférica. 2. Asimetría izquierda en una tarea de reproducción verbal». Revista de Psicologia General y Aplicada, 1982, 37, 4, 637-646.

MANning, L., y FERnÁndeZ-Ballesteros, R.: "Tactile perceptual task and field dependence». Trabajo

- presentado en la Sesión de Poster del Third European Meeting on Cognitive Neuropsychology. Bressanone, enero, 1985.

MAnning, L.; Fernández-Ballesteros, R., y MAcí́, A.: «Validez racional de algunas pruebas de dependencia de campo». En prensa.

MiLNER, B.: "Laterality effects in audition». En V. B. Mouncastle (Ed.) Interbemispberic relations and Central Dominance. John Hopkins Press, Baltimore, 1962.

Oltman, Ph.; Ehrlichman, H., y Cox, P.: «Field independence and laterality in perception of faces». Perceptual and Motor Skills, 1977, 45, 255-260.

Pizzamiglio, L.: «Handedness, ear preference and field dependence». Perceptual and Motor Skills, 1974, 38, 700-702.

Pizzamiglio; L., Zoccolotti, P., Mammucari, A., y Cesaroni, R.: «The independence of face identity and facial expression recognition mechanisms: Relationship to sex and cognitive styles». Brain, 1983, 2, 176-188.

Witkin, H.; Goodenough, D., y Oltman, Ph.: «Psychological differentiation current status. Journal of Personality and Social Psycbology, 1979, 37, 7, 1127-1145.

ZoccolotT1, P., y Oltman, Ph.: «Field dependence and lateralization of verbal and configurational processing». Cortex, 1978, 14, 155-163.

ZoccolotT1, P.; PASSAFIUme, D., y Pizzamiglio, L.: «Hemispheric superiorities on a unilateral tactil test: Relationship to cognitive dimensions». Perceptual and Motor Skills, 1979, 49, 735-742. 